\title{
Safety Evaluation on Interventional treatment for Gynecological Disease
}

\author{
Yanli LV \\ People's Hospital of Zhengzhou, Zhengzhou 450000, Henan Province, P.R.China \\ email: tuke126@126.com
}

Corresponding AuthorL Yanli Lv

Keywords: Gynecological disease, Interventional treatment, Safety, Evaluation.

\begin{abstract}
This study is to detect and evaluate the safety of interventional treatment in gynecological disease. Method: Interventional treatment was performed on 43 cases of gynecological patients. The surgery time information was statistically collected. the collateral circulation establishment was investigated, along with the pathological changes in myometrium after embolization and X-ray radiation dose during operation. Result: the operation mean time was (40.3 \pm 6.1$)$ minutes; few necroses happened in endometrium ( $2 / 17$ cases), shallow myometrium (12/17 cases); for X-ray radiation dosage during the operation were all within the safety range in normal condition: sacrum projection for $32.88 \mathrm{cGy}$, ovary for $16.64 \mathrm{cGy}$ and paranephros projected waist skin area for $12.44 \mathrm{cGy}$; ovarian artery supplied stream blood collateral circulation to uterus, which was detected by ovary DSA radiography and 3-D color Doppler imaging. Conclusion: the interventional treatment was a safe operation for gynecological disease.
\end{abstract}

\section{Introduction}

The interventional therapy has extensively developed in many gynecological field. Good results had been achieved, while, there was no denying that some problems had appeared in the process of implementation of interventional treatment[1 3]. In order to gain a safely application and technology, the interventional treatment for gynecological disease had been systematically evaluated on safety since 2004 in People's Hospital of Zhengzhou.

\section{Material and method}

\section{Study subject}

From March 2004 to March 2014, there were a total of 267 cases of patients with gynecological disease who had performed interventional therapy. 43 cases of patients were chosen as the research object. The age ranged from 24 years old to 66 years old, average was $43.7 \pm 14$.1years old. In the 26 cases of benign disease, there were 15 cases of uterine fibroids and 11 cases with adenomyosis; 17 cases patients were all suffering cervical malignant tumors ( 15 cases for II stage, 2 cases for IIIa stage). The X-ray radiation dosage were detected among the 26 cases of patients with benign disease; pathology examination were performed on the uterine specimens from 17 cases of cervical cancer patients after interventional therapy.

\section{Method}

\section{1) Interventional treatment method}

Selding's technology were employed to complete the femoral artery catheter. 4.0 5.0F Cobra-2 catheter was based on the disease condition to finalize the internal iliac artery embolization (IIAE) or uterine arterial embolization (UAE). Antibiotics or anticarcinogen were injected during the operation. Embolization agent were the fresh genlatin sponge granule or PVA.

\section{2) Pathological observation}

The radical surgery were utilized on cervical cancer patients in the $14 \sim 21$ days after interventional therapy. For the removed uterine specimens, the endometrium, myometrium, ovarian, fallopian tube were respectively collected, and fixed in 10\% formalin solution, then dehydrated conventionally, embedded by paraffin in order to gain serial section, thickness was around $5 \mu \mathrm{m}$, and 
then HE staining were performed.

\section{3) $\mathrm{X}$-ray radiation dosage}

For the 26 cases of patients with benign disease, the thermoluminescent dosimeter (TLD) were respectively set at the area of eyebrow, thyroid, xiphoid, one inch below umbilicus, skin area of bilateral adrenal waist projection, the corresponding skin area of sacrococcygeal region and postoperative vaginal vault area before operation, and immediately removed after surgery. The radiation dose at postoperative vaginal vault area was set as the ovarian radiation dose.

\section{Observation}

\section{1) Operation time}

Operation time was calculated from the femoral artery puncture to catheter pull-out.

\section{2) Pathological changes after interventional therapy}

Optical microscope were applied to observe the collected specimens. The main observation included: cell morphology complete condition, cytoplasm dyeing depth, nucleus shape ( presence of pyknosis, disintegration, dissolution and disappear), etc.

\section{3) $\mathrm{X}$-ray radiation dosage detection}

FJ-427A type micro-computerized thermal absorption dosimeter were applied to detect the specific value.

\section{4) Embolism collateral circulation after surgery}

DSA image were achieved through DSA imaging dynamic observation, especially the blood supply after the embolization of bilateral iliac artery and uterine artery.

\section{Statistical method}

Mean Standard Deviation.

\section{Result}

\section{Surgery time}

47 interventional surgery were performed on the 43 cases of patients, surgery time was 30 35 minutes, average was $40.3 \pm 6.1$ minutes, the surgery time of different disease was shown in table 1 .

Table 1. Interventional surgery time of different disease

\begin{tabular}{|l|l|l|l|}
\hline Name & $\begin{array}{l}\text { Case quantity } \\
(\mathrm{n})\end{array}$ & $\begin{array}{l}\text { Time range } \\
(\mathrm{min})\end{array}$ & $\begin{array}{l}\text { Average } \\
\text { time }(\mathrm{min})\end{array}$ \\
\hline gynecological malignant tumor & 17 & $34 \sim 35$ & $43.6 \pm 6.0$ \\
\hline Benign gynecological tumor & 26 & $30 \sim 51$ & $37.5 \pm 5.5$ \\
\hline
\end{tabular}

\section{Pathological changes}

Pathological examination were employed to detect the specimens from the 17 cases of cervical cancer after radical surgery. The optical microscope detection results of endometrium, myometrium, overian, fallopian tube were below: endometrium: most cases ( $12 / 17)$ were shown edema, few (2/17) showed small range of local necrosis, some (3/17) showed large area of necrosis; myometrium: partly cases (12/17) with uterus shallow muscularis showed small area of necrosis, focal coagulation necrosis, discontinuity focal necrosis, mainly near the endometrium, obvious necrosis were not seen in muscularis and plasma muscle; obvious pathological changes were not seen in ovary and fallopian tube.

\section{$\mathrm{X}$-ray radiation dosage in intraoperative patients}

The details of X-ray radiation dosage of 26 cases of gynecology intraoperative detection as shown in table 2 . 
Table $2 \mathrm{X}$-ray radiation dose of ovarian and other area for intraoperative patients

\begin{tabular}{|l|l|}
\hline Detection Area & X-ray absorbed dose (cGy) \\
\hline eyebrow & 0.008 \\
\hline thyroid & 0.009 \\
\hline xiphoid & 0.05 \\
\hline one inch below umbilicus & 0.86 \\
\hline skin area of left adrenal waist projection & 12.04 \\
\hline skin area of right adrenal waist projection & 12.44 \\
\hline sacrococcygeal region & 32.88 \\
\hline ovary & 16.64 \\
\hline
\end{tabular}

\section{Collateral circulation}

After UAE treatment, the 5 cases of patients were immediately implemented right ovarian artery intubation. The right ovarian artery supplied the blood stream to uterus, and shown the uterine partly development.

\section{Discussion}

Interventional therapy was the main part of the interventional radiology, which widely used in the treatment of various disease, and achieved good results. In the treatment of gynecological disease, as a relatively new treatment, it was significant to evaluate the safety and stability of interventional treatment. As the intervention surgery was under the X-ray radiation, catheter technology was applied to block the vascular blood flow of the target organ in order to achieve embolization. For gynecology disease, it need to embolizate the uterine artery, the uterus artery blood supply. So the safety assessment should include: surgery time, endometrium, myometrium, ovarian, fallopian tube, the pathological changes after embolization, X-ray radiation of intraoperative patients and establishment of collateral circulation.

\section{Surgery time}

Surgery time was the significant indicator of safety to measure gynecology interventional treatment. On one hand, surgery time was positive proportional related to X-ray radiation received during operation, on the other hand, although a part of gynecological disease cases belongs to elective surgery, quite a number of emergency situation belongs to hemorrhage of malignant tumor and uterine adenomyosis. As the time-consuming operation time could aggravate the patients disease situation. In general, intervention surgery time was significantly shorter than traditional hysterectomy surgery [4]. In the paper, the statistical information told the shortest is 30 minutes, the longest time was 55 minutes, average $40.3 \pm 6.1$ minutes. In the process, the taken of DSA image four times need a certain time, so the actual operation time was much shorter than the time.

\section{Intraoperative $\mathrm{X}$-ray irradiation for patients}

Whether X-ray radiation received by intraoperative patients could cause harm to patients, expecially the impact on ovary was the issue concerned by crowds. As interventional surgery should be performed under DSA, in order to gain better and qualified images, this study employed the intervention radiation traditional situation, and directly, it directly attracted patients the X-ray radiation dosage of patients. The excessive radiation dosage in the operation could cause the radiation injury. The X-ray directly illuminated the pelvis of patients. The women with gynecological disease were mostly child-bearing age patients, ovarian follicles in the differentiation development unceasingly, and more sensitive to radiation. Therefore, the detection of X-ray radiation received in the interventional treatment should cause enough attention. Different tissues and organs had different sensitivity to radiation, and had a tolerance range. Once exceeded the range, it will cause damage. According to the literature report [5], 1\% 5\% patients could develop ovarian injury within 5 years, when the ovaries was under the radiation dose of $200 \sim 300 \mathrm{cGy} ; 25 \% \sim 50 \%$ patients could develop ovarian injury within 3 years, when the ovaries was under the radiation dose of 2000 3000cGy; Skin ratiotherapy dose was 5500cGy. This study measured interventional treatment of ovarian radiation dose was $16.64 \mathrm{cGy}$, the radiation dose of sacrococcygeal region skin 
area $32.88 \mathrm{cGy}$, which was obviously lower than the injury dose of overies and skin. In the follow-up of 3 8 month after surgery, 26 patients with benign disease had normal menstruation. Therefore, the radiational dose of gynecological interventional treatment on overian, skin and other organs were in the normal tolerance range, and could not cause obvious damage.

\section{Female reproductive organ pathological changes and blood flow changes after embolization}

Uterus was a kind of solid organ, the embolism of the main blood supply artery got embolism could cause corresponding necrosis, which had no doubt. But in the literature review had been observed that there was few serious necrosis in the uterus. In order to extensively under the pathological changes of uterus, ovaries, fallopian tubes, 17 cases of patients with ovarian cancer patients who receive radical surgery after $14 \sim 21$ days of interventional surgery. The above pathological changes were statistically detected. There were only few mild shallow uterine muscular layer or scattered coagulation necrosis. 3 cases patients with serious large areas of endometrium necrosis, 2 cases with mild necrosis. This demonstrated uterus could still get blood supply through other collateral circulation to support the basic demand, after the embolization of bilateral uterine artery. In order to prove this part, right ovarian artery catheter imaging after bilateral uterine artery embolization, the uterus could still have blood supply through other blood stream to maintain the basic demand. The 3-D color photograph 24 hours after surgery also confirmed it. The blood flow is less. Ovary artery was one of the six major transportation. There were also other communicating brunch participating in blood supply. In addition, the embolization agent used in surgery was also crucial, the fresh gelfoam particles or larger diameter of PVA particle were chosen, which blocked the larger blood vessels and terminal in order to enable uterine body retain complete capillary network. Other communicating brunch could supply small amount of blood to the uterus through capillary network, in order to make sure that uterine not serious necrosis. When uterine artery got embolization, the ovary could provide enough blood supply to maintain the function of the ovaries. So, there was no obvious change of pathology, fallopian tubes and ovaries had sufficient communicating brunches also could get enough blood supply, and not cause changes in pathology.

\section{Summary}

From the above study, interventional treatment for gynecological disease was safe, and would not cause in patients with severe complications. But there were still some problems to be solved, such as to further shorten the operation time, to reduce the DSA intake times in surgery, to reduce the DSA imaging condition in order to decrease the X-ray radiation intake dosage of patients in surgery, which was the further direction of this study.

\section{References}

[1] Chunming Chen, Lizhi Liang, Peiming Liu, et al, Clinical research on interventional treatment of gynecological malignant tumor in middle-late stage, Chinese Journal of Practical Gynecology and Obstetrics,vol.11, pp.667, 2000.

[2] Yanjun Guol Zhonghua Shi, The complications of interventional treatment on gynecological malignant tumor. Chinese Journal of Radiology, Vol.5, pp.332, 2001.

[3] Mingpei Liu, Chunming Chen, Diagnosis and treatment on gynecological interventional treatment of thrombogenesis of external iliac artery. Chinese Journal of Practical Gynecology and Obstetrics, Vol.12(supplementary issue), pp.130, 1996.

[4] Chunming Chen, Xiaomao Li, Guoliang Li, et al. Application research of thrombotic interventional therapy on severe postpartum hemorrhage, Chinese Journal of Practical Gynecology and Obstetrics, Vol.2, pp.84, 2001.

[5] Zeyi Cao, et al. Gynecological Oncology. Beijing: Beijing Publishing House, pp.458, 1999. 